\title{
Abdominal wall reconstruction with reconstructive tissue matrix
}

\author{
I Bombil, B Puttergill, BS Ngutshane \\ Department of Surgery, Chris Hani Baragwanath Academic Hospital, University of the Witwatersrand
}

Corresponding author: I Bombil (bombil@telkomsa.net)

\begin{abstract}
Summary: A giant desmoid tumour required full thickness excision of the lower abdominal wall. The resultant massive defect was closed with reconstructive tissue matrix (Strattice ${ }^{\mathrm{TM}}$ ). The mesh acted as successful scaffold for regenerative repair enabling skin grafting of a stable granulation bed giving a satisfactory mechanical and cosmetic result. This mesh should be explored further as a simpler alternative to complex plastic reconstructive techniques for large abdominal soft tissue defects.
\end{abstract}

S Afr J Surg 2018;56(3)

http://dx.doi.org/10.17159/2078-5151/2018/v56n3a2430

\section{Case report}

A 29-year-old female patient presented with a two-year history of a giant tumour of the anterior abdominal wall. The tumour, which was attached to the bladder dome, required a full thickness resection of almost all of the anterior abdominal wall to obtain clear surgical margins, except at the site where it was shaved off the pubic bone.
The resultant massive defect (Figure 1a) was bridged with Reconstructive Tissue Matrix (Strattice ${ }^{\mathrm{TM}}$ ) (Figure 1b) which is a surgical mesh derived from porcine skin and is processed and preserved in a phosphate buffered aqueous solution containing matrix stabilisers. A vacuum assisted closure (VAC) which was changed every three days was used to protect the mesh and allow granulation of the defect. After

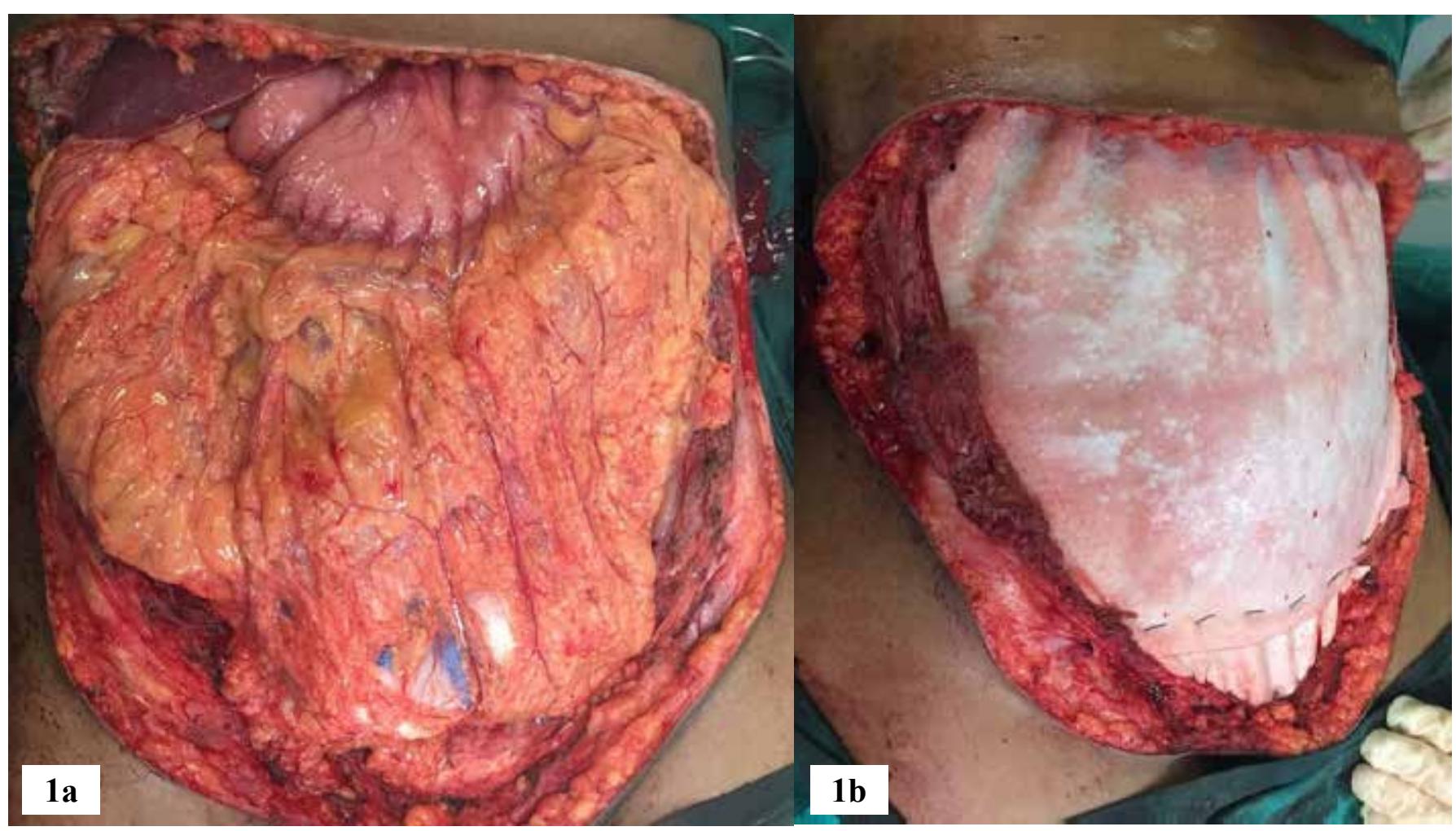

Figure 1 a Massive abdominal wall defect post tumour resection and $\boldsymbol{b}$ Defect bridged with Strattice ${ }^{\mathrm{TM}}$ 


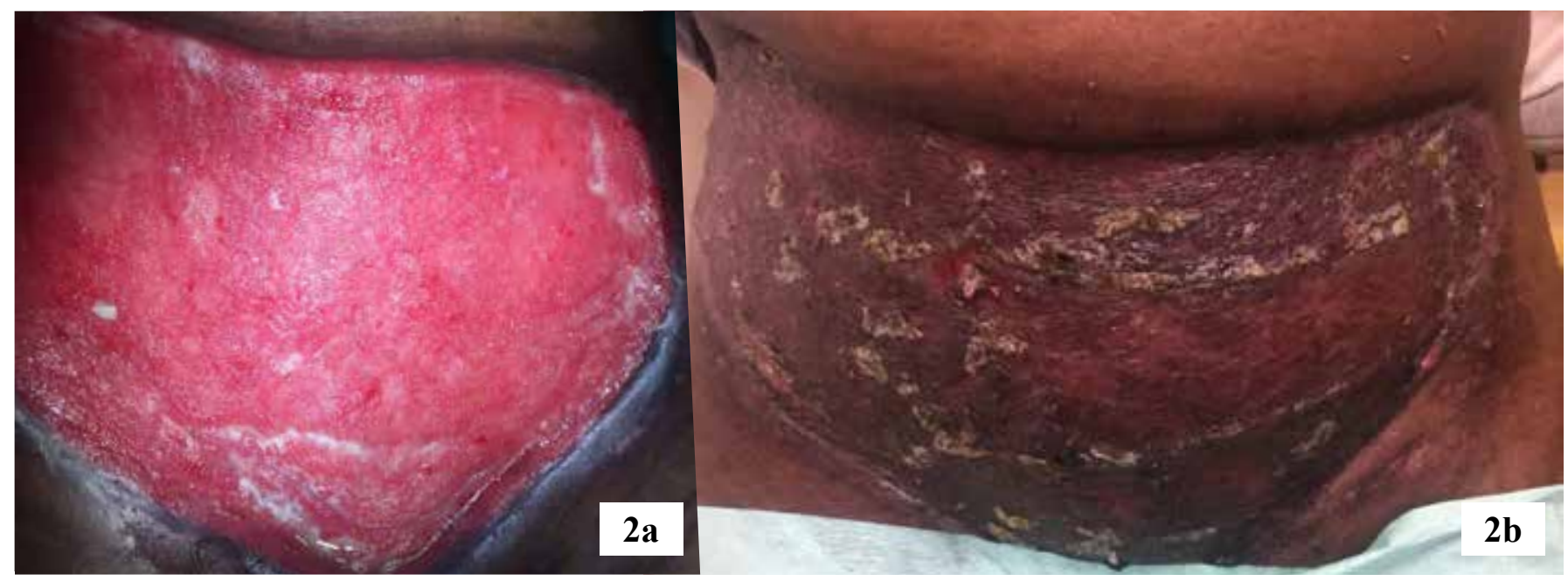

Figure 2 a Granulation bed after 6 weeks and $\boldsymbol{b}$ Healed SSG.

5 weeks, the Strattice ${ }^{\mathrm{TM}}$ was fully covered by the granulation tissue (Figure 2a). The split skin graft performed a week later was successful (Figure 2b). The patient was discharged uneventfully on the 7 th week.

The ethic approval (clearance certificate No. M170295) was obtained from the Human Ethics Committee of the University of the Witwatersrand and by the Research Review Board of Chris Hani Baragwanath Academic Hospital.

\section{Discussion}

Abdominal wall reconstruction of large defects remains a challenge. Various options are available to reconstruct these defects. Myofascial release and component separation are not applicable in this patient since the myofascial complex was excised. The anterolateral thigh fasciocutaneous flap, the tensor fascia lata pedicled flap, and the whole thigh flap have all been described but they are more suitable for smaller defects. ${ }^{1}$ Synthetic meshes were entertained as a possible option, but their use in this case without skin cover is subject to higher risk of mesh sepsis, fistula formation and adhesive bowel obstruction ${ }^{2}$ and led us to the choice of the only remaining alternative - a biological implant. We needed a fascial substitute to cover the intra-abdominal viscera and to provide a base for granulation tissue and for a subsequent split skin graft to survive. Strattice ${ }^{\mathrm{TM}}$ is designed to cover soft tissue defects while presenting a scaffold for cellular infiltration neovascularisation and regenerative repair. ${ }^{3}$

Strattice successfully simplified the management of this complex case, obviating the need for difficult, prolonged flap reconstruction that was contra-indicated in this exceptional case.

\section{REFERENCES}

1. Koshy CE; Kumar MV; Evans J. Lower abdominal wall reconstruction using the anterior thigh fasciocutaneous flap. $\mathrm{Br}$ J Plast Surg. 1999; 52(8):667-9 (ISSN: 0007-1226)

2. Servant JM; Arnault E; Revol M. Reconstruction of large thoracoabdominal defects using two-stage free tissue transfers and prosthetic materials. J Plast Reconstr Aesthet Surg. 2006; 59(4):360-5 (ISSN: 1748-6815)

3. Michael J. Rosen, MD; David M. Krpata, MD; Bridget Ermlich, RN; Jeffrey A. Blatnik, MD. A 5-Year Clinical Experience With Single-Staged Repairs of Infected and Contaminated Abdominal Wall Defects Utilizing Biologic Mesh. Annals of Surgery. 2013;257(6):991-996. 